\title{
Post-inflation increase of the cosmological tensor-to-scalar perturbation ratio
}

\author{
N. Bartold* \\ The Abdus Salam International Centre for Theoretical Physics, Strada Costiera 11, 34100 Trieste, Italy \\ Edward W. Koll $\dagger$ \\ Particle Astrophysics Center, Fermi National Accelerator Laboratory, Batavia, Illinois 60510-0500, USA \\ and Department of Astronomy and Astrophysics, Enrico Fermi Institute, \\ University of Chicago, Chicago, Illinois 60637-1433, USA
}

A. Riott团

Department of Physics and INFN Sezione di Padova, via Marzolo 8, I-35131 Padova, Italy

(Dated: December 14, 2005)

\begin{abstract}
We investigate the possibility that the amplitude of scalar density perturbations may be damped after inflation. This would imply that CMB anisotropies do not uniquely fix the amplitude of the perturbations generated during inflation and that the present tensor-to-scalar ratio might be larger than produced in inflation, increasing the prospects of detection of primordial gravitational radiation. It turns out, however, that the damping of density perturbations is hard to achieve.
\end{abstract}

PACS numbers: 98.80.cq

Inflation [1] has become the dominant paradigm for understanding the initial conditions for structure formation and for Cosmic Microwave Background (CMB) anisotropies. In the inflationary picture, primordial density and gravitational-wave fluctuations are created from quantum fluctuations, "redshifted" beyond the horizon during an early period of superluminal expansion of the universe, then "frozen" 2, 3]. Perturbations at the surface of last scattering are observable as temperature anisotropies in the CMB, as first detected by the Cosmic Background Explorer satellite [4, 5]. The last and most impressive confirmation of the inflationary paradigm has been recently provided by data from the Wilkinson Microwave Anisotropy Probe (WMAP) satellite, which marks the beginning of the precision era of CMB measurements in space [6]. The WMAP collaboration has produced a full-sky map of the angular variations of the CMB to unprecedented accuracy. WMAP data support the inflationary mechanism as the mechanism for the generation of super-horizon curvature fluctuations.

The amplitude of cosmological perturbations on large scales can be expressed in terms of a gauge-invariant variable, $\zeta$, describing density perturbation on uniformcurvature slices [3]: $\zeta=-\psi-H \delta \rho / \dot{\rho}$, where the density perturbation $\delta \rho$ and the curvature perturbation $\psi$ are defined on a generic slicing, $H=\dot{a} / a$ is the Hubble rate ( $a$ is the scale factor), and the dot stands for differentiation with respect to cosmic time, $t$. If the pressure perturbation is non-adiabatic, the comoving curvature perturbation $\zeta$ may evolve on super-horizon scales as [7]

$$
\dot{\zeta}=-\frac{H}{\rho+P} \delta P_{\text {nad }},
$$

where $P$ is the pressure of the fluid and $\delta P_{\text {nad }}=\delta P-$ $(\dot{P} / \dot{\rho}) \delta \rho$ is the non-adiabatic pressure.
In the standard slow-roll scenario associated with single-field models of inflation, the observed density perturbations are due to fluctuations of the inflaton field as it slowly rolls along its potential $V(\phi)$. When inflation ends, the inflaton $\phi$ oscillates about the minimum of its potential and decays, thereby reheating the universe. As a result of the fluctuations, each region of the universe goes through the same history, but at slightly different times. The final temperature anisotropies are caused by the fact that the duration of inflation was different in different regions of the universe, leading to adiabatic perturbations.

It is usually assumed that $\zeta$ remains constant on superhorizon scales, so the curvature perturbation $\zeta$ calculated shortly after Hubble exit $(k=a H)$ during inflation determines the curvature perturbation until that scale re-enters the Hubble radius during the subsequent radiation- or matter-dominated phase. Therefore, in the conventional inflationary scenario, the amplitude of the comoving curvature perturbation generated during inflation may be directly related to the large-scale temperature anisotropies through the Sachs-Wolfe relation $\delta T / T=-(1 / 5) \zeta$. The power spectrum of curvature perturbations in slow-roll inflationary models is predicted to be [1]

$$
\mathcal{P}_{\zeta}(k)=\frac{k^{3}}{2 \pi^{2}}\left|\zeta_{\mathbf{k}}\right|^{2}=\frac{1}{2 M_{p}^{2} \epsilon}\left(\frac{H_{*}}{2 \pi}\right)^{2}\left(\frac{k}{a H_{*}}\right)^{n_{\zeta}-1},
$$

where $n_{\zeta} \simeq 1-2 \eta+6 \epsilon$ is the scalar spectral index, $\epsilon=$ $\left(\dot{\phi}^{2} / 2 H^{2} M_{p}^{2}\right) \simeq\left(M_{p}^{2} / 2\right)\left(V^{\prime} / V\right)^{2}$ and $\eta=M_{p}^{2}\left(V^{\prime \prime} / V\right)$ are the slow-roll parameters, $M_{p}=(8 \pi G)^{-1 / 2} \simeq 2.4 \times$ $10^{18} \mathrm{GeV}$ is the reduced Planck mass, and $H_{*}$ indicates the Hubble rate during inflation.

The generation of gravitational waves (tensor perturbations) is another generic prediction of an accel- 
erated de Sitter expansion of the universe. Gravitational waves, whose possible observation might come from the detection of the $B$-mode of polarization in the CMB anisotropies [8], may be viewed as ripples of spacetime around the background metric $g_{\mu \nu}=d t^{2}-$ $a^{2}(t)\left(\delta_{i j}+h_{i j}\right) d x^{i} d x^{j}$. The tensor $h_{i j}$ is traceless and transverse and has two polarizations $(\lambda=+, \times)$. Since gravitational-wave fluctuations are (nearly) frozen on superhorizon scales, a way of characterizing them is to compute their spectrum on scales larger than the horizon.

In the conventional slow-roll inflationary models where the fluctuations of the inflaton field $\phi$ are responsible for the curvature perturbations, the power spectrum of the gravitational waves is $\mathcal{P}_{T}(k)=\left(k^{3} / 2 \pi^{2}\right) \sum_{\lambda}\left|h_{\mathbf{k}}\right|^{2}=$ $\left(8 / M_{p}^{2}\right)\left(H_{*} / 2 \pi\right)^{2}\left(k / a H_{*}\right)^{n_{T}}$, with $n_{T}=-2 \epsilon$. Since the fractional changes of the power spectra with scales are much smaller than unity, one can safely consider the power spectra as roughly constant on the scales relevant for the CMB anisotropy and define a tensor-to-scalar amplitude ratio

$$
r=0.86 \frac{\mathcal{P}_{T}}{\mathcal{P}_{\zeta}}=13.7 \epsilon \simeq-6.8 n_{T} .
$$

This expression provides also a consistency relation between $r$ and the tensor tilt $n_{T}$. The present WMAP dataset yields the upper bound $r \lesssim 0.5[9]$. Since the scale of inflation in slow-roll models is fixed to be $V_{*}^{1 / 4} \simeq 3.7 r^{1 / 4} \times 10^{16} \mathrm{GeV}$, in order to match the observed amplitude of CMB anisotropies one can already infer an upper bound on the energy scale of inflation of about $4 \times 10^{16} \mathrm{GeV}$. The corresponding upper bound on the Hubble rate during inflation, $H_{*}$, is about $4 \times 10^{14}$ $\mathrm{GeV}$. A positive detection of the tensor modes through the $B$-mode of CMB polarization (once foregrounds due to gravitational lensing from local sources have been properly treated) requires $r \gtrsim 10^{-3}$, corresponding to $V_{*}^{1 / 4} \gtrsim 3 \times 10^{15} \mathrm{GeV}$ and $H_{*} \gtrsim 2 \times 10^{12} \mathrm{GeV}[10]$.

Let us now consider inflation-model phenomenology in the light of these considerations [1]. Large-field models of inflation characterized by power-law potentials $V \propto \phi^{p}$ give a value of $r=7\left(1-n_{\zeta}\right) p /(p+2)$, making gravitational waves potentially detectable. However, for smallfield models of inflation with potentials of the type $V \simeq$ $V_{0}\left[1-(\phi / M)^{2}\right]$, one has $r \simeq 3.5\left(1-n_{\zeta}\right) e^{-\left(1-n_{\zeta}\right) N} \lesssim 1 / N$ which is undetectable ( $N$ is the number of $e$-folds); for potentials of the form $V \simeq V_{0}\left[1-(\phi / M)^{p}\right]$, with $p>2$, one has $r \simeq 7 p^{2}\left(M / M_{p}\right)^{2 p /(p-2)}[N p(p-2)]^{-(2 p-2) /(p-2)}$, which is detectable only if $M$ is much larger than $M_{p}$. Similar results hold if $V$ is a mixture of terms, say quadratic at small $\phi$ and quartic at larger $\phi$, provided that all terms have the same sign. Hybrid models of inflation, where the inflaton field is accompanied by another field responsible for most of the potential energy, also typically have small $r$.

Another way of posing the same problem was suggested in Ref. 11]. The slow-roll paradigm gives, using the defi- nition of $\epsilon$ and Eq. (3), $|d \phi / d N| / M_{p}=(r / 6.9)^{1 / 2}$, where $d \phi$ is the change in the inflaton field in $d N=H d t \simeq d \ln a$ Hubble times. The range of scales corresponding to the relevant multipoles in the $\mathrm{CMB}$ anisotropy corresponds to $\Delta N \simeq 4.6$, and therefore the field variation is $\left(\Delta \phi / M_{p}\right) \simeq(r / 0.2)^{1 / 2}[10]$. This is a minimum estimate because inflation continues for some number $N$ of $e$-folds of order of 50. The detection of gravitational waves requires in general variation of the inflaton field of the order of the Planck scale [1], and it is therefore difficult to construct a satisfactory model of inflation firmly rooted in modern renormalizable particle theories. There is, therefore, a theoretical prejudice against the likelihood of observation of gravitational-wave detection within slow-roll models of inflation where the curvature perturbation is due to the fluctuations of the inflaton field itself.

This picture sounds fairly pessimistic. The detection of gravitational waves requires large values of $r$, but the large majority of single-field models of inflation are characterized by the hierarchy $\epsilon \ll \eta$, and therefore by tiny values of $r$. The pessimism relies, however, on the standard assumption that the comoving curvature perturbation $\zeta$ remains constant on large scales from the end of the inflationary epoch down to the matter-dominated era. The goal of this Letter is to raise the possibility that, within well motivated particle physics scenarios, the amplitude of the curvature perturbation may be damped during the evolution of the Universe after inflation. If this is possible, the amplitude of CMB anisotropies on large scales does not immediately fix the normalization of the comoving curvature perturbation generated during inflation, $\zeta_{\text {inf }}=-\psi-H \delta \phi / \dot{\phi}$. In other words, the comoving curvature perturbation on large scales at the matter-dominated epoch, $\zeta_{\mathrm{m}}$, is not equal to the curvature perturbation during inflation, but it is smaller by a damping factor $\delta<1$ :

$$
\zeta_{\mathrm{m}}=\delta \zeta_{\mathrm{inf}}
$$

What might be the origin of this damping? The total curvature perturbation is the weighted sum of the individual perturbations, $\zeta_{i}=-\psi-H \delta \rho_{i} / \dot{\rho}_{i}$, associated with the various fluids present in the Universe:

$$
\zeta=\sum_{i} \frac{\dot{\rho}_{i}}{\dot{\rho}} \zeta_{i}
$$

If after inflation the energy density of the Universe is dominated by some fluid " $F$ " other than radiation, and this fluid is not perturbed, then $\zeta_{\mathrm{F}}=0$ and the total curvature perturbation is given by $\zeta \simeq\left(\dot{\rho}_{\gamma} / \dot{\rho}_{\mathrm{F}}\right) \zeta_{\gamma} \simeq$ $\left(\rho_{\gamma} / \rho_{\mathrm{F}}\right) \zeta_{\text {inf }}$, where we have identified the curvature perturbation of radiation $\zeta_{\gamma}$ with the curvature perturbation $\zeta_{\text {inf }}$ generated during inflation. When the fluid $\mathrm{F}$ decays into relativistic degrees of freedom commencing conventional radiation domination, the total curvature perturbation (and therefore the CMB anisotropies) on 
large scales is damped with respect to the curvature perturbation generated during inflation by a factor

$$
\delta \sim\left(\rho_{\gamma} / \rho_{\mathrm{F}}\right)
$$

computed at the decay time. In simple words, the damping arises because the fluid releases entropy during its decay, but does not change the perturbations since $\zeta_{\mathrm{F}}=0$. Therefore, the amplitude of CMB anisotropies on large scales does not uniquely fix the normalization of the comoving curvature perturbation generated during inflation, and the energy scale of inflation would be fixed by CMB measurements to be

$$
V_{*}^{1 / 4} \simeq 7.1 \frac{\epsilon^{1 / 4}}{\delta^{1 / 2}} \times 10^{16} \mathrm{GeV} .
$$

This energy can be rather large even in single-field models where the slow-roll parameter $\epsilon$ is tiny, thanks to the damping factor $\delta<1$.

An important fact is that gravitational waves are not similarly damped on super-horizon scales. The theoretically predicted value of the tensor-to-scalar amplitude ratio would then be larger than what is commonly predicted using Eq. (3),

$$
r=0.86 \frac{\mathcal{P}_{T}}{\mathcal{P}_{\zeta}}=13.7 \frac{\epsilon}{\delta^{2}} \simeq-6.8 \frac{n_{T}}{\delta^{2}} .
$$

Correspondingly, the consistency relation of Eq. (3) is violated and the field variation of the inflaton field is given by $\left(\Delta \phi / M_{p}\right) \simeq \delta(r / 0.2)^{1 / 2}$. The parameter $r$ can be within the detectability range even in those singlefield models of inflation, such as small-field models and hybrid models, where otherwise the detection would be a hopeless task. Moreover the detection of gravitational waves would not require embarrassing variations of the inflaton field as large as the Planckian scale.

Let us now discuss the possible source of the damping factor. Suppose that, beyond the inflaton field, there is another (set of) complex scalar field(s), generically denoted by $\Phi$. For instance, in the context of either supergravity or superstring theories, many models of supersymmetry breaking predict the presence of particles with Planck-suppressed couplings to ordinary matter [12]. These particles are generically called moduli. (Warped) Calabi-Yau compactifications possess a large number of moduli, such as complex structure moduli, Kähler moduli, and dilaton moduli, corresponding to the deformations of the compact manifold. Flux compactifications can classically stabilize the moduli [13, 14], and their masses $m_{\Phi}$ are typically much larger than the weak scale 15. This also holds in scenarios such as split supersymmetry [16], in which the scale of supersymmetry breaking is orders of magnitude larger than the weak scale and moduli are expected to acquire masses between $10^{10}$ and $10^{13} \mathrm{GeV}$.
Let us first analyze the case of those moduli which receive a large mass during inflation because of the supersymmetry breaking driven by the vacuum energy driving inflation 12]. Barring cancellations, one expects that the mass of $\Phi$ would be larger than the Hubble rate during inflation. For example, a supergravity Kähler potential of the type $\delta K=\left(\Phi^{\dagger} \Phi \phi^{\dagger} \phi / M_{p}^{2}\right)$ induces a mass squared for $\Phi$ equal to $3 H_{*}^{2}$. For the same reason, a modulus is expected to be displaced from its presentday minimum. Because of its large mass, the quantummechanical fluctuations of the field $\Phi$ generated during inflation, $\zeta_{\Phi}=-\psi-H \delta \rho_{\Phi} / \dot{\rho}_{\Phi}$, can be safely taken to vanish. After the end of inflation, when the Hubble parameter becomes smaller than $m_{\Phi}$, the field $\Phi$ rolls toward its present value and oscillates about it with an initial amplitude $\Phi_{0}$, approximately equal to the distance between the minima of the effective potential at large $H$ during inflation and small $H$ after inflation. During oscillations the energy density of the modulus $\Phi$ scales like non-relativistic matter, and is therefore expected to dominate rather quickly over the radiation energy density produced during reheating after inflation. At later times the modulus will decay into relativistic degrees of freedom.

However, no damping of the comoving curvature perturbation can occur in this case. The reason is that the modulus starts to oscillate in different parts of the universe when the local value of the Hubble rate is equal to $m_{\Phi}$. But there are local variations in $H$ due to $\zeta_{\text {inf }}$, and consequently a nonvanishing $\zeta_{\Phi}=\zeta_{\text {inf }}$ will be induced. To see it more concretely, one can analyze the equations for the background modulus field $\Phi$ and for its fluctuation $\delta \Phi$

$$
\begin{aligned}
\ddot{\Phi}+3 H \dot{\Phi}+V^{\prime} & =0, \\
\ddot{\delta \Phi}+3 H \dot{\delta \Phi}+V^{\prime \prime} \delta \Phi & =4 \dot{\Phi} \dot{\psi}-2 V^{\prime} \psi,
\end{aligned}
$$

where we have assumed that the potential can be expanded linearly, $\delta V \sim V^{\prime} \delta \Phi$. The modulus $\Phi$ may start moving either when the universe is still dominated by the energy stored in inflaton oscillations (with equation of state $w \simeq 0$ ) or after reheating when the energy density is mainly due to the thermal bath (with equation of state $w \simeq 1 / 3$ ). In both cases, the gravitational potential $\psi$ can be considered to be constant. The solution of Eq. (9) then reads $H \delta \Phi \simeq(2 / 3(1+w)) \dot{\Phi} \psi$. This leads to the initial condition $\zeta_{\Phi}=-(5+3 w) /(3(1+w)) \psi=\zeta_{\text {inf }}$ when the modulus starts oscillating, and no non-adiabatic pressure is available to change the total comoving curvature perturbation [17].

Let us consider, in turn, those moduli which typically do not receive a large mass during inflation, the stringy axions [18]. If the Kähler potential does not depend upon the imaginary part of the modulus field, call it $\sigma$, then the mass of the axion-like field may be exponentially suppressed and the condition $m_{\sigma}^{2} \ll H_{*}^{2}$ does not require 
any particular fine-tuning [19]. The field $\sigma$ is quantummechanically excited during inflation, $\delta \sigma \sim\left(H_{*} / 2 \pi\right)$. However, if the scale $f_{\sigma}$ appearing in the periodic potential $V(\sigma)$ is smaller than $H_{*}$, then the linear approximation of the fluctuation of the potential $V(\sigma)$ fails and $\delta \rho_{\sigma}$ is basically zero. This is because any long-wavelength fluctuation of the axion field $\sigma$ does not change the average distribution of $V(\sigma)$ [20], thus suppressing $\delta V$. This suppression due to the non-linearities of the potential leads to

$$
\delta \rho_{\sigma} \simeq 0
$$

at the beginning of the radiation phase after inflation. The suppression of this entropy perturbations is basically due to the fact that the perturbations do not follow the background because of the non-linear potential. However, again, the background value of the $\sigma$ field will start oscillating around the minimum of the potential at different times in different separated Hubble volumes because of the presence of the adiabatic perturbations generated by the inflaton field. Therefore, adiabatic initial conditions will be inherited by the field $\sigma$ leading again to $\zeta_{\sigma}=\zeta_{\gamma}$.

While the cases analyzed in this short note lead to a negative conclusion, it would be most interesting to see if there are (less obvious) sources of damping of the primordial curvature perturbation generated during inflation.

We would like to thank M. Sloth for very useful correspondence about his related paper [18] and for readily pointing out an error in the first version of our draft. We thank A. Linde, D. Lyth, S. Mukhanov L. McAllister, M. Sasaki and D. Wands for useful comments and criticisms. E.W.K. is supported in part by NASA grant NAG5-10842 and by the Department of Energy.

* Electronic address: nbartolo@ictp.trieste.it

† Electronic address: rocky@fnal.gov
‡ Electronic address: antonio.riotto@pd.infn.it

[1] D. H. Lyth and A. Riotto, Phys. Rept. 314, 1 (1999).

[2] V. F. Mukhanov and G. V. Chibisov, JETP Lett. 33, 532 (1981)

[3] J. M. Bardeen, P. J. Steinhardt, and M. S. Turner, Phys. Rev. D 28, 679 (1983).

[4] C. L. Bennett et al. Astrophys. J. 464, L1 (1996).

[5] K. M. Gorski et al. Astrophys. J. 464, L11 (1996).

[6] C. L. Bennett et al., Astrophys. J. Suppl. 148, 1 (2003).

[7] J. M. Bardeen, Phys. Rev. D 22, 1882 (1980).

[8] M. Kamionkowski, A. Kosowsky and A. Stebbins, Phys. Rev. Lett. 78, 2058 (1997); U. Seljak and M. Zaldarriaga, Phys. Rev. Lett. 78, 2054 (1997).

[9] H. V. Peiris et al., Astrophys. J. Suppl. 148, 213 (2003); W. H. Kinney, E. W. Kolb, A. Melchiorri and A. Riotto, Phys. Rev. D 69, 103516 (2004).

[10] M. Kesden, A. Cooray and M. Kamionkowski, Phys. Rev. Lett. 89 (2002) 011304; L. Knox and Y.-Song, Phys. Rev. Lett. 89 (2002) 011303.

[11] D. H. Lyth, Phys. Rev. Lett. 78, 1861 (1997).

[12] G. D. Coughlan, W. Fischler, E. W. Kolb, S. Raby and G. G. Ross, Phys. Lett. B 131, 59 (1983); A. S. Goncharov, A. D. Linde and M. I. Vysotsky, Phys. Lett. B 147, 279 (1984). B. de Carlos, J. A. Casas, F. Quevedo and E. Roulet, Phys. Lett. B 318, 447 (1993) [arXiv hep-ph/9308325].

[13] S. Kachru, R. Kallosh, A. Linde and S. P. Trivedi, Phys. Rev. D 68, 046005 (2003) [arXiv hep-th/0301240].

[14] O. DeWolfe, A. Giryavets, S. Kachru and W. Taylor, arXiv hep-th/0505160

[15] O. DeWolfe and S. B. Giddings, Phys. Rev. D 67, 066008 (2003) [arXiv hep-th/0208123.

[16] G. F. Giudice and A. Romanino, Nucl. Phys. B 699, 65 (2004) [Erratum-ibid. 706, 65 (2005)]; N. Arkani-Hamed and S. Dimopoulos, arXiv hep-th/0405159

[17] Moduli fields may be produced also by thermal scatterings during reheating with a number density normalized with respect to the entropy density $s: n_{\Phi} / s \sim$ $\left(T_{\mathrm{RH}} / M_{p}\right) T^{3}$. The curvature perturbation of these thermally produced moduli will be also equal to $\zeta_{\gamma}=\zeta_{\text {inf }}$.

[18] M. S. Sloth, arXiv hep-ph/0507315

[19] T. Banks and M. Dine, Nucl. Phys. B 505, 445 (1997).

[20] L. A. Kofman and A. D. Linde, Nucl. Phys. B 282, 555 (1987). 